Priv.-Doz. Dr. med. Thomas P. Hüttl

Ärztlicher Direktor

Chefarzt der Abt. f. Allgemein- und Viszeralchirurgie

Chirurgische Klinik München-Bogenhausen

Prof. Dr. med. Karl-Walter Jauch (Foto)

Direktor der Chirurgischen Klinik und Poliklinik der LMU München - Klinikum Großhadern
29_ Internistische Aspekte

32- Chirurgische Methoden

\title{
Adipositaschirurgie
}

\section{Mit dem Skalpell gegen Übergewicht und Diabetes}

— Spätestens bei fortgeschrittener Adipositas versagen konservative Therapieansätze nahezu regelhaft. Hoffnungen auf medikamentöse Ansätze waren bislang vergeblich, die verfügbaren Substanzen wenig effektiv und inzwischen nicht mehr zugelassen.

\section{Vom dicken Dicken zum dünnen Dicken}

Seit geraumer Zeit wird der Übergewichtskrankheit mit beachtlichem Erfolg laparoskopisch durch spezielle adipositaschirurgische Verfahren an die Pfunde gegangen. Dabei liegt der Verlust an Übergewicht_nicht zuletzt in Abhängigkeit vom gewählten Verfahren, zwischen 50 und 70\% in den ersten zwei Jahren. Auch der Überlebensvorteil und eine verbesserte Lebensqualität dürfen inzwischen als gesichert angesehen werden. Die Chirurgie ist jedoch keine kausale Lösung. Gene und andere Umweltfaktoren bleiben unverändert, der „dicke Dicke“ wird zum „dünnen Dicken“. Der einstmals vorhande Übergewichtszustand würde analog des Jo-Jo-Efektes nach konservativen Therapien wieder erreicht werden, würden dies nicht Magenband oder Magenrestriktion mit und ohne Malabsorption (Schlauchmagen, Magenbypass) verhindern. Auch schafft die Chirurgie neue Risiken wie Mangelerscheinungen oder chirurgische Langzeitkomplikationen wie Narbenbrüche, Band- und Portkomplikationen. Langfristig nachhaltige Erfolge jenseits von drei bis fünf Jahren mit zudem geringen Nebenwirkungen erfordern deshalb neben einer Umstellung der Lebensgewohnheiten eine individuell angepasste interdisziplinäre Mindestbegleittherapie durch adipositaserfahrene Ärzte und Therapeuten.
Hoch spezialisierte Zentren mit guter interdisziplinärer Zusammenarbeit wie in München im Klinikum Großhadern und an der Chirurgischen Klinik MünchenBogenhausen, sind eher selten. Sie reichen für eine flächendeckende Versorgung dieses in den nächsten Jahren um den Faktor Zehn auf 25000-35000 Eingriffe pro Jahr ansteigenden chirurgischen Krankengutes in keinster Weise aus.

\section{Interdisziplinäre Konzepte erforderlich}

Ziel der beiden Übersichtsarbeiten ist es, Ihnen einen Überblick über die chirur$\odot^{* 0^{k 0}}$ gischen Möglichkeiten und deren Ergebnisse zu geben, dabei aber auch die hausärztlich, internistisch und ernährungsmedizinisch relevanten Probleme und deren Lösungsmöglichkeiten aufzuzeigen. Praktikable prä- und postoperative Mindeststandards werden ebenso vorgestellt wie Überlegungen zur „metabolischen Chirurgie“.

Die American Diabetes Association hat in diesem Zusammenhang vor wenigen Monaten die chirurgischen Operationen wie Magenband und Magenbypass in das Spektrum der empfehlenswerten Therapieoptionen bei schlecht einstellbarem Diabetes auch ohne extremes Übergewicht (BMI unter $35-40 \mathrm{~kg} / \mathrm{m}^{2}$ ) aufgenommen.

Das medizinische Problem der fortgeschrittenen morbiditären Fettleibigkeit ist jedoch mit dem Skalpell allein nicht beherrschbar. Interdisziplinäre Konzepte in Zusammenarbeit mit chirurgischen Experten sind gefordert. Grundkenntnisse auch in der chirurgischen Therapie und Nachsorge dieser Volkskrankheit sind deshalb von jedem Arzt und Therapeuten zu fordern. 